\title{
Utilidad en educación musical de las aplicaciones móviles informáticas de Android y Apple: estudio comparativo
}

Adrián Cantos Soler ${ }^{1}$

\section{Resumen}

Los recursos electrónicos como metodología didáctica son una posibilidad y, cada vez más, una realidad en muchos centros educativos. Incluso puede llegar a ser una necesidad en determinados casos específicos de alumnos o alumnas con necesidades educativas específicas que, gracias a la tecnología y a las aplicaciones móviles informáticas, pueden mejorar notablemente su rendimiento y progreso intelectual.

Palabras clave: Apps educativas, TIC, didáctica de la música, Android y Apple, educación musical.

\section{Abstract}

The electronic resources as a didactic methodology are a possibility and, increasingly, a reality in many educational centers. It may even become a necessity in certain specific cases of students with specific educational needs who, thanks to technology and mobile computer apps, can significantly improve their performance and intellectual progress.

Keywords: Educational App's, ICT, didactics of music, Android and Apple, musical education.

\section{Introducción}

Este estudio tiene como objetivo comparar las aplicaciones educativas musicales gratuitas y de pago en móviles y tabletas con sistema operativo Android y Apple para conocer cuáles pueden resultar más útiles en el ámbito educativo. Además, se propone un posible modelo a seguir para evaluar las aplicaciones, tomando decisiones en base a los resultados obtenidos.

También se tendrán en cuenta las pizarras digitales usadas en las aulas de los centros escolares, las cuales utilizan el sistema operativo del ordenador al que estén conectado, pudiendo ser Linux, Windows o Apple.

\footnotetext{
${ }^{1}$ IES Josep Lluís Sert, Castelldefels, Barcelona. Artículo recibido: 11 de octubre de 2017; aceptado: 20 de noviembre de 2017.
} 
En la sociedad de la tecnología y la información en la que vivimos, el uso cotidiano y constante de las herramientas tecnológicas parece ser imprescindible. Verdaderamente son muy útiles y facilitan en gran medida la labor educativa, son una herramienta de innovación y que aporta aire fresco a las metodologías, sobre todo a las más tradicionales, pero en ningún caso deberían llegar a considerarse imprescindibles.

En este ámbito, la educación no ha quedado relegada a un segundo plano, por lo que los recursos electrónicos se van implantando, no sin polémica, en la metodología docente en el aula.

Si bien es cierto que se han llevado a cabo estudios sobre la forma de evaluar el software educativo, sobre todo pensado para aquel que se utiliza en los ordenadores de sobremesa de los centros educativos, no se han elaborado sobre aquel que se encuentra disponible para otro tipo de soportes como son los móviles o las tabletas.

Quizás sea debido a la polémica generada cuando se piensa en incluirlos en la metodología diaria del aula. Así pues, un gran número de veces, la controversia es creada por docentes que no han utilizado las TIC [Tecnología de la Información y la Comunicación], mostrándose reticentes a hacerlo en un futuro. Algunos de ellos han opinado en las encuestas realizadas que las aplicaciones educativas de música serían más una distracción que un elemento de aprendizaje.

\section{Marco teórico}

Sobre el tema que nos ocupa, tal vez por su actualidad, escasea la bibliografía. No obstante, podemos citar algunos trabajos como ejemplos de estudios en los que se valora la experiencia educativa mediante el uso de soportes móviles. Dichas investigaciones tienen en común que son estudios de caso centrados en el uso de la tableta de Apple, el iPad.

Ebner, Schönhart y Schön [2014] exponen los resultados sobre el aprendizaje móvil en niños de entre 6 y 10 años. Algunas de las conclusiones a las que han llegado son que: se les han de hacer juegos fuera de la aplicación pero relacionados con ésta, de forma que se potencie la creatividad;se han de preparar comentarios inmediatos a su utilización para establecer una retroalimentación entre las aplicaciones y los usuarios y que las aplicaciones han de servir para trabajar en equipo, no como fomento de la competitividad.

El iPad es un dispositivo que, pese a ser de muy fácil manejo, con la cada vez más abundante oferta de aplicaciones, en ocasiones se hace complicado encontrar la app correcta (Rudolph y Leonard, 2014].

En el estudio realizado por Bostock [1998] se tuvo en cuenta, entre otros aspectos, la interacción que se produce entre usuario y aplicación, es decir, el feedback. 
De entre todos los estudios realizados en torno a las nuevas tecnologías, innovación e incursión o adaptación a los espacios, es especialmente destacable la labor y contribución de Pere Marquès [2007]. Pere Marquès, profesor titular jubilado de la Universidad Autónoma de Barcelona, es especialista en la aplicación de nuevas metodologías didácticas para mejorar los procesos de enseñanza y aprendizaje con la ayuda de las TIC, así como en el diseño, desarrollo y evaluación de recursos multimedia para la educación.

La ficha de catalogación que éste propone como modelo de evaluación, y que nos ha servido de guía para nuestra propuesta, contempla dos vertientes: pedagógica y funcional por un lado y técnica por otra. Cada uno de ellos es evaluado por una serie de características e indicadores que se valoran a través de una escala cualitativa [Marquès, 2002].

De forma genérica, si tomáramos las marcas de los sistemas operativos como autores, en función de que son éstos los que proporcionan todas las apps, serían dos a los que haríamos referencia: Android y Apple. No obstante, como autores se citarán a los desarrolladores y/o creadores de las aplicaciones educativas.

Seguiremos líneas referenciales que se centrarán, sobre todo, en artículos de investigación que han sido fruto de ponencias en congresos o en publicaciones de revistas digitales de diferentes universidades, tanto nacionales como extranjeras. También nos será de gran utilidad la lectura y análisis de algunos blogs dedicados a dar consejos y proponer ideas novedosas en educación, así como artículos digitales de docentes y expertos en la materia.

De entre todas, podemos citar a la Universidad de Salamanca, a la Universidad de La Rioja, a la Universitat Oberta de Catalunya, a la Universidad de Oviedo, a la Universidad de Navarra, a la Universidad Nacional del Chaco Austral [Argentina], a la Universidad de Guadalajara de Jalisco [México] y a la Universidad Abierta de Lisboa [Portugal] como ejemplos de instituciones que han investigado sobre el tema que abordamos.

\section{Metodología}

Pasando a otro aspecto y dado que hablar de cada uno de los conceptos técnicos de las tecnologías educativas se nos escaparía del ámbito de estudio de este artículo, nos centraremos en las aplicaciones interpretadas, es decir, aquellas que se pueden descargar desde la App Store [tienda de aplicaciones]e instalar en nuestros dispositivos electrónicos. Éstas son más económicas que las Apps Nativas y reportan una mejor experiencia que las Web Apps.

Hemos confeccionado una ficha de evaluación de apps educativas con la finalidad de poder contrastarlas lo más clara y eficazmente posible, de forma que con un simple vistazo podamos obtener una rápida conclusión sobre si nos conviene utilizarla en el aula o no. 
Hemos seguido el ejemplo de algunos modelos elaborados por estudiosos del tema como Pere Marquès [2005] o Consuelo Belloch [2013], pero también basándonos en aquellos aspectos que más valoran de una aplicación los alumnos/as y los docentes mediante los resultados de las encuestas realizadas.

Nuestra propuesta de evaluación ha sido pensada para valorar aplicaciones móviles, por lo que los apartados que constarán en la ficha serán:

Descripción. Se dividirá en cuatro subsecciones: Objetivos, Contenidos, Función que cumple la aplicación [informar, educar, etc.] y Nivel Educativo al que va orientado.

Gráficos. Sería equivalente a la interfaceen terminología informática, se dividirá en 4 subsecciones: Sencillez, Claridad expositiva, Comodidad y Calidad. Esta última subdividida a su vez en: Audio, Video y Elementos en pantalla.

La Sencillez hace referencia a la cantidad de elementos que nos aparece en pantalla, siendo valorada como muy sencilla aquella que no tenga muchos elementos, a lo que irá asociado una sensación más intuitivita. La Claridad expositiva hace referencia a la organización de dichos elementos, siendo evaluada como muy clara aquella que se presente de forma ordenada y que fácilmente podamos entender cómo funciona. La Comodidad hace referencia a lo que también podríamos denominar como entorno amable, es decir, que no se producen redirecciones a páginas o aplicaciones de terceros o que no contiene molestos anuncios de publicidad. La Calidad de Audio se refiere a si los sonidos empleados son realistas, en caso de aplicaciones que simulan instrumentos musicales; de Video a que éstos se visualizan correctamente y el contenido es apropiado; y los Elementos en pantalla se refiere a la calidad de los detalles.

El punto Jugabilidad se divide en: Flexibilidad, que hace referencia a la capacidad de cambiar la dificultad y los contenidos que se trabajan, y Estructura por niveles, es decir, si se va aumentado progresivamente la dificultad conforme se van asimilando los conocimientos de las lecciones o tareas de la app.

La Pedagogía se divide en cuatro puntos: Integración con el nivel educativo de los alumnos a los que va dirigido, es decir, si se corresponde correctamente con los usuarios que la van a utilizar; Elementos motivacionales, como podrían ser recompensas en forma de bonificaciones o mejoras de algún tipo (siempre en un entorno virtual y responsable); Tutorización, es decir, si se presta a ser manejada de forma regulada por el docente, como herramienta en la que se plantean tareas y los alumnos/as las realizan, sin poder continuar hasta que el profesor no considere. 0 si, por el contrario, la aplicación permite libre disposición a los alumnos/as para explorar e investigar todas las posibilidades de la misma sin control del profesor. Y Recursos, donde se valorará si se utilizan esquemas, explicaciones mediante vídeo de aquello que se trabaja, imágenes, sonidos asociados al contenido, etc. para potenciar la asimilación de contenidos. 
La Metodología se subdivide en: Prueba-error, si se procede mediante la selección de diferentes respuestas posibles o contestación personalizada, como podría suceder en las aplicaciones de reconocimiento auditivo de acordes o notas; Programación de las tareas, es decir, posibilidad de organización sincronizada con el calendario de las tareas a realizar; y Evaluación global o por actividades en función de si la app puntúa al finalizar toda la serie de tareas o de forma individual y separada cada una de ellas.

En Extras se subdivide en: Instrucciones, valorándose si se incorpora algún tutorial o manual de utilización de las diferentes funciones que ofrece la app; Exportación/Importación,donde se valorará que la app permita exportar/importar múltiples formatos y compartir el trabajo que hemos realizado; Información del desarrollador, útil en el caso que se quiera contactar por cualquier incidencia.

En Observaciones se podrán hacer todo tipo de sugerencias y comentarios de aquellos aspectos que no se han tratado en la ficha y que se consideren relevantes, como el precio o el sistema operativo, y en Valoración global se hará una recapitulación de todos los puntos evaluados y se estimará la viabilidad de la app, así como su relación calidad-precio, u otros enfoques que se consideren oportunos.

\section{Resultados}

Todas las aplicaciones educativas cumplen con una serie de características comunes:

Ser didácticas.

$>$ Poder ser utilizadas en soportes móviles como teléfonos inteligentes [smartphones] $\mathrm{y} / \mathrm{o}$ tabletas (tablets).

> Ser interactivas, produciéndose un feedback entre alumno/a y/o profesor/a, y el dispositivo electrónico.

Son de uso principalmente individual pero, mediante actividades de colaboración e interacción grupal, pueden ser usadas en conjunto gracias al carácter flexible.

Uso sencillo, ya que no se requieren conocimientos previos de informática. Ahora bien, cada app puede tener unas directrices de uso distintas que serán necesarias conocer. Aun así, el tiempo que llevará a docentes y alumnos/as hacerse con el control de la aplicación será escaso.

Esta serie de características será aplicable también a los programas educativos [software] en equipos de sobremesa. 


\subsection{Resultados de las muestras totales de apps.}

Dado que hemos realizado una comparativa de dos sistemas operativos diferentes con las variables gratuitas y de pago, el número total de aplicaciones varía ligeramente.

Aunando las que son gratuitas y las que son de pago, el total de aplicaciones que hemos detallado en Apple son 150, mientras que en Android asciende a 159.

Así pues, el número total de aplicaciones gratuitas en Android han sido de 128, contra las 51 de pago. Por otra parte, en el otro sistema operativo estudiado, iOS Apple, el número total de aplicaciones gratuitas suman 71, contra las 88 que figuran como de pago.

Más concretamente, en Android hay un total de 22 aplicaciones que se encuentran tanto en formato gratuito como de pago, predominando las clasificadas como aptas para Secundaria/ Bachiller. Por el contrario, en Apple solo existen 9 que se encuentren en ambos formatos, predominando el nivel Infantil.En el iOS Apple predominan las aplicaciones de pago [55\%] y en Android las de tipo gratuito [72\%] [Ver fig. 1].

Fig. 1. Porcentajes de aplicaciones gratuitas y de pago en Apple y Android.

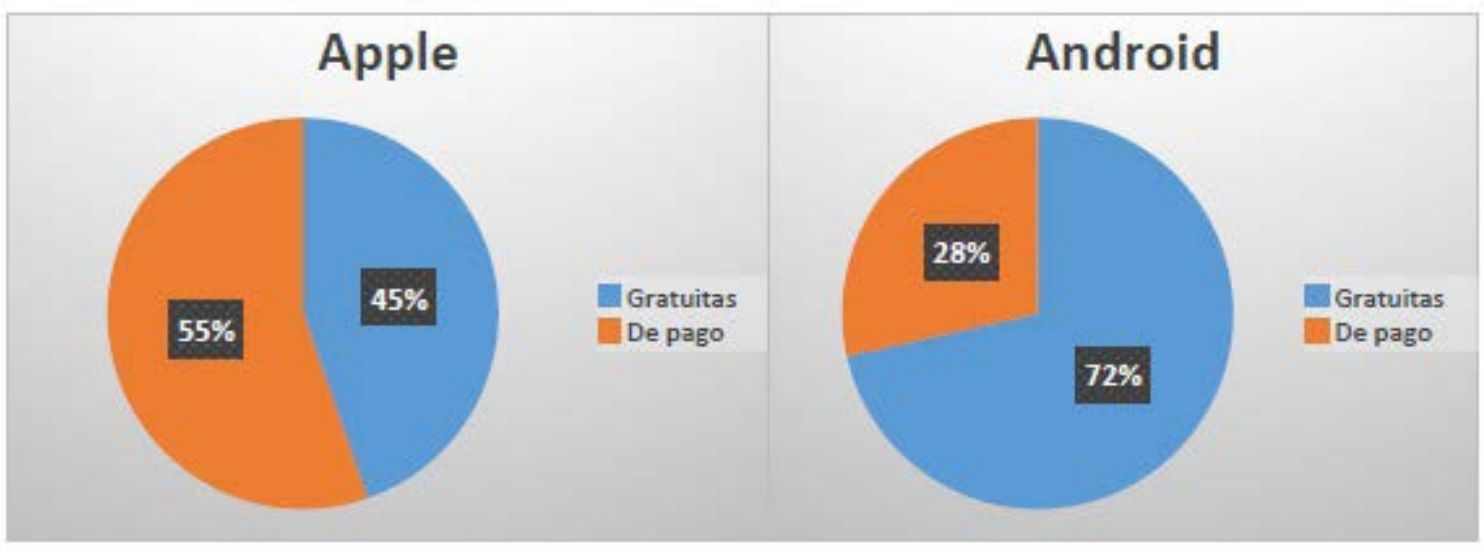

\subsection{Resultados de las encuestas.}

Los resultados revelan que han sido los docentes los que más han participado [Fig. 2].

Fig. 2. Resultados de las encuestas ${ }^{2}$

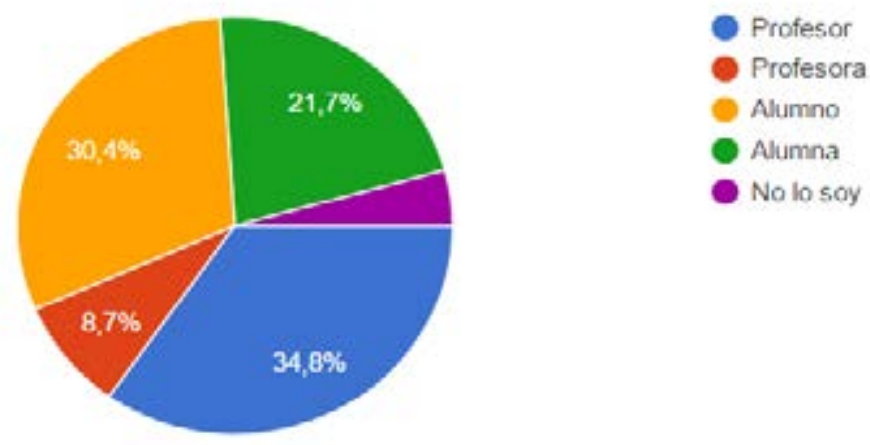

${ }^{2}$ Extraído de las respuestas generadas de nuestra encuesta mediante Google Forms. 
A la pregunta de si alguna vez uno de los profesores de música había hecho uso de alguna app, los resultados son que el 42,9\% no han utilizado nunca ninguna app y el 57,1\% sí que lo han hecho, encontrándose entre ellas MuseScore, Metronome, Spotify y Tunable.

En cuanto al soporte utilizado para interactuar con las apps, se impone el teléfono móvil con el 47,4\% [Fig. 3).Un dato significativo y representativo es el sistema operativo usado por los encuestados, ya que el más usado ha sido Android con un 57,1\% [Fig. 4].

Fig. 3. Porcentajes de soportes electrónicos

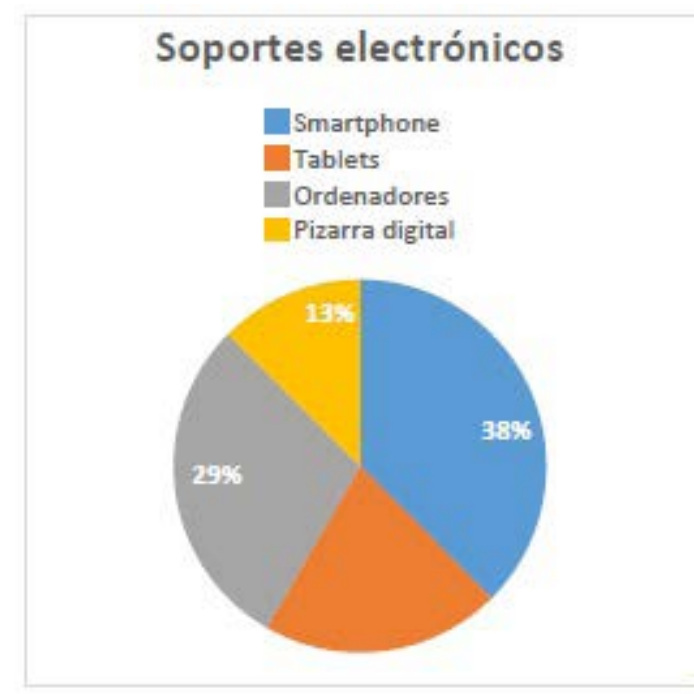

Fig.4. Porcentajes de sistemas operativos.

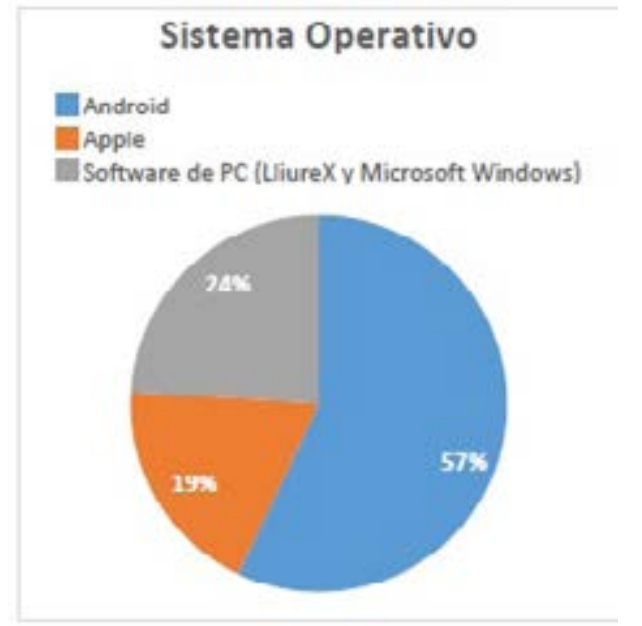

Un $86.6 \%$ de los usuarios que nunca han utilizado una app en la clase de música han opinado que sí que hubiera sido útil su uso, argumentando que hubiera propiciado un aprendizaje más productivo, complementando al realizado en clase, aportando dinamismo y facilitando la labor explicativa al docente y de comprensión al alumno/a. 
Como respuesta a la polémica en torno al uso de apps en el aula, el 81.8\% de los encuestados han coincidido en opinar, no sin matices, que su utilización sería más beneficiosa que perjudicial. Dichos matices se pueden resumir en que serán beneficiosas siempre y cuando se planifique su uso, controlando el tiempo que se le dedica y su justificación pedagógica. En cambio, el 18.2\% opina que también hay desventajas en su inclusión en el aula, como por ejemplo que no todos los alumnos/as pueden permitirse tener un dispositivo electrónico [sobre todo tabletas] y que pueden propiciar distracción.

En relación al uso de apps en casos de alumnos/as con DTA, un 64.2\% no ha hecho uso nunca de una aplicación como metodología didáctica, argumentando alguno de ellos que no ha sido necesario, tan solo una mayor atención y conocimiento de cómo tratar el tipo de trastorno. Así, el 35.8\% sí que ha utilizado una app en soporte tableta o pizarra digital, nunca Smartphone.

El 93.75\% sí que emplearía una app en el caso que alguna vez se le presentara la casuística de un DTA, argumentando que es un recurso que puede facilitar el aprendizaje gracias a que son intuitivas. El 6.25\% opina que no las emplearía porque, entre otros motivos, siempre es mejor y más natural la "enseñanza directa".

Existe unanimidad en que las apps contribuyen a la mejora de las dolencias en el ámbito educativo, ya que se pueden trabajar los mismos contenidos pero desde varias perspectivas: juegos visuales, centrando la atención, creando vínculos de interés entre ellos/as, etc.

\subsection{Apps destacadas para Secundaria.}

De entre toda la amplia oferta de aplicaciones musicales que podemos encontrar en las tiendas de Android y Apple, las que a continuación exponemos resultan de gran utilidad en el aula de música de Secundaria.

\subsubsection{Escribir música}

En Android, la app NotateMeNow cumple con su propósito de ofrecernos un soporte electrónico donde escribir nuestra música. Esta aplicación cuenta con una versión gratuita de prueba [demo] y con otra de pago, la cual tiene un precio de 35'99 $€$.

En Apple también la podemos encontrar, por lo que con esta app podríamos trabajar en ambos sistemas operativos. No obstante, la versión de pago en este sistema operativo es un poco más caro que en Android, estando en 39'99 € su precio.

Esto en cuanto a aplicaciones que podemos encontrar tanto gratis como de pago. Ahora bien, 
existen alternativas de mejor calidad pero que solo están disponibles pagando, como pueden ser Notiono Scorio Music Notator. Ambas se encuentran disponibles en la plataforma Apple, siendo Notion la más completa de todas ellas.

No obstante, la opción más interesante sería Ensemble Composer, ya que es totalmente gratuita y permite exportar por correo electrónico partituras creadas en formato MIDI y XML. Esta opción nos permitirá programar ejercicios a realizar en clase y en casa con tan solo compartir los archivos. Además, el formato XML es compatible con otros editores de partituras como MuseScore, por lo que las facilidades son mayores.

\subsubsection{Practicar ritmo y solfeo}

Con Ritmo y solfeo [Gamya] podremos practicar los conceptos básicos hasta los avanzados en la lectura del ritmo musical en dispositivos Android. Además, viene con un reto online que te permite medir tus progresos con respecto a otros usuarios. Esta aplicación también se encuentra de pago, siendo su precio 0'99€.

Si en nuestra aula de música hay batería, una aplicación que nos será de gran utilidad será Leer música para Batería [Pablo Prieto]. Dicha app se encuentra disponible únicamente en el sistema operativo Apple, habiendo una versión gratuita y una de pago con un precio de 2'29€. La versión gratuita incluye setenta lecciones y setenta tareas, pudiendo practicar diferentes estilos musicales como Pop Rock, Blues Rock, Jazz, Latin, Funk o Fusion.

Aplicación similar existe en Android, con el mismo nombre pero del desarrollador Apps Musycom. La versión de pago tiene un precio de 0’99 €.

\subsubsection{Visualizar partituras}

Si queremos tener un soporte donde poder visualizar las partituras que vayamos a trabajar en el aula, podemos descargarnos de forma gratuita la aplicación del editor de música MuseScore. Con ella vamos a poder escuchar la partitura, seleccionar las secciones que se muestren y se reproduzcan o transportar la melodía, entre otras.

Esta aplicación tiene una versión de pago, Cancionero MuseScore, siendo su precio 2'39 €. Lo que nos va a permitir la versión pro va a ser abrir archivos MuseScore [.mscz] vía Dropbox, correo electrónico o navegador.

En iOS, piaScore - Smart Music Score Reader, nos permitirá descargarnos partituras del IMSLP y visualizarlas en nuestro dispositivo de forma fácil y cómoda. Esta app es gratuita.

\subsubsection{Instrumentos virtuales}

Una de las ventajas más interesantes de utilizar apps en el aula es poder descubrir y experimentar nuevas sonoridades, conocer instrumentos que físicamente sería muy difícil o imposible. 
En Android, la aplicación Walk Band - Estudio de Música nos permitirá tocar con xilófonos, piano, batería y muchos más instrumentos. Además, incluye un modo de juego en el que dos alumnos/ as podrán tocar simultáneamente desde el mismo dispositivo. Una ventaja más, es totalmente gratis.

En Apple, la más conocida y valorada de todas las aplicaciones de instrumentos virtuales es Garage Band. Esta aplicación tiene un precio de 4'99 € pero es una de las más completas que existen, ya que cuenta con muchísimos instrumentos, desde pianos de gran cola, pasando por pianos eléctricos, claves, sintetizadores, ensembles de cuerda y metales, clarinetes, flauta, guitarras acústicas y eléctricas, lira, etc. Además de batería, caja de ritmos y un batería de estudio virtual con más de 30 ritmos de diferentes estilos musicales.

En definitiva, una gran variedad de instrumentos para un sinfín de posibilidades.

Si únicamente queremos practicar xilófono, en Android podremos utilizar la app Professional Xylophone. Ésta es gratuita, aunque existe otra que es de pago, Professional Xylophone Elite, con un precio de 0'99€.

En Apple tenemos la posibilidad de descargarnos Xylophone Orff [Timothy Purdum]. Es una app de pago con un precio de 0'99 €, la cual permite tocar con los cinco instrumentos del conjunto de xilófonos Orff: Bajo, Contralto, Alto, Soprano y Sopranino o Glockenspiel. Sobre las láminas se disponen las letras de las notas para facilitar el aprendizaje, estando disponibles las notas Sib y Fa\#.

\section{Conclusiones}

De forma posterior al análisis y estudio de los resultados obtenidos, llegamos a las conclusiones siguientes.

La plataforma Apple cuenta con más aplicaciones de pago que Android, lo que puede significar un hándicap importante en su incursión en el aula.

Otra conclusión es que Android cuenta con más app's por las que no hay que no pagar ni un solo euro, pero también es cierto que dichas app's gratuitas pueden ser muy limitadas en su uso, ya que son de muestra[también denominadas demo]. Estas aplicaciones son una versión incompleta, de prueba y evaluación para el usuario, con el fin de que vea y testee la idea y posibilidades que ofrece dicha app.

Así pues, este tipo de app's tiene sus ventajas e inconvenientes, ya que puedes probar antes de pagar la app pero, en el caso que sea útil, no dispones de casi ninguna opción interesante. Esto en Apple no sucede tan a menudo, ya que o se encuentran gratuitas con todas las posibilidades desbloqueadas o se encuentran de pago, no hay término medio. 
En cuanto a las encuestas, llegamos a las siguientes conclusiones:

Más de la mitad del profesorado de música ha hecho uso en el aula de alguna aplicación mediante un teléfono móvil Android, siendo Kahoot una de las más habituales. Seguido de los dispositivos móviles encontramos el software educativo de los ordenadores de sobremesa, usados también mediante las pizarras digitales, como Audacity o MuseScore.

Concluimos que los docentes coinciden en que el uso de aplicaciones educativas en el aula sería una acción beneficiosa, incluso aquellos que no han hecho uso de esta herramienta de forma pedagógica, argumentando que propiciaría un aprendizaje más productivo, complementando al realizado en clase, aportando dinamismo y sirviendo para captar la atención de los alumnos/as, desarrollando más la faceta práctica y, en definitiva, facilitando la labor explicativa al docente y de comprensión y asimilación al alumno/a.

En contraposición, la problemática que existe es que no todos los alumnos pueden permitirse comprar un dispositivo electrónico, a lo que proponemos como posible solución que se haga cargo el centro educativo mediante subvenciones ${ }^{2}$ disponibles, entre otras vías, de la adquisición de dichos dispositivos. Sabemos que confluyen muchos factores distintos y que no siempre será viable, por lo que será una muy buena alternativa trabajar con las Web Apps en ordenadores o pizarras digitales.

Las funciones que han de cumplir las aplicaciones móviles para que puedan llegar a considerarse educativas son: trabajar contenidos acordes al nivel educativo en el que se van a emplear, generar un proceso en el que se asuman e interioricen los conocimientos y habilidades, proveer grados de dificultad en los que se refleje el progreso en el aprendizaje y ser motivadoras, incentivando la pedagogía y metodología activa.

En cuanto al uso de las aplicaciones educativas en alumnos/as que padecen algún tipo de dificultad de aprendizaje, concluimos que sería acertado utilizar dichas herramientas gracias a su carácter intuitivo,proporcionándoles facilidades en su proceso de aprendizaje y adquisición de los conocimientos a trabajar.

Además, contribuye de forma positiva a la mejora en la recuperación, en los casos en que es posible, del déficit.

No obstante, pese al gran consenso existente, todavía se hace carente la puesta en práctica de dichas aplicaciones educativas.

\footnotetext{
${ }^{3}$ Como la reciente Resolución de 30 de mayo de 2017, del director general de Política Educativa, por la que se convocan subvenciones y asignaciones económicas para desarrollar proyectos de investigación e innovación educativa en centros docentes sostenidos con fondos públicos de la Comunitat Valenciana, durante el curso académico 2017-2018. [DOCV 8060].
} 


\section{Referencias}

Alcantud, F., Ávila, V., Martínez Martínez, R., Romero, R. [2001]. Estudio sobre el impacto de las nuevas tecnologías en las personas con discapacidad. Valencia: Universitat de València. Recuperado de http://roderic.uv.es/bitstream/handle/10550/23762/InformeFinal. pdf?sequence $=1$

Barroso, J., Valverde, J., Medel, J. L. [1998]. Evaluación de medios informáticos: una escala de evaluación para software educativo. En M. Cebrián de la Serna [Coord.], Creación de materiales para la innovación educativa con nuevas tecnologías. Málaga: Universidad de Málaga, Instituto de Ciencias de la Educación.

Bostock, S. [1998]. Evaluating checklist. Evaluating training software. Lancaster: Lancaster University.

Brown, A. [2014]. Music technology and education: Amplifying musicality. Brisbane, Australia: Routledge.

Cabero, J. [2000]. Nuevas tecnologías aplicadas a la educación. Barcelona: Síntesis.

Cataldi, Z. (2000). Metodología de diseño, desarrollo y evaluación de software educativo [Tesis de Magister en Informática, Universidad Nacional de La Plata, Argentina]. Recuperado de http:/ / www.iidia.com.ar/rgm/tesistas/cataldi-tesisdemagistereninformatica.pdf

Cova, A., Arrieta, X. [2008). Modelo de evaluación de software educativo en el área de la física [Tesis doctoral inédita]. Universidad del Zulia, Maracaibo, Venezuela.

Cova, A., Arrieta, X., Aular de Duran, J. [2008]. Revisión de modelos para evaluación de software educativo. Revista Electrónica de Estudios Telemáticos 7 [1], 1-21. Recuperado de https:// dialnet.unirioja.es/descarga/articulo/2954394.pdf

Dickens, H. J., \& Churches, A. [2011]. Apps for learning: 40 best iPad/iPod Touch/iPhone apps for high school classrooms. Vancouver, Canada: CorwinPress.

Dorrego, E. [1998). Modelo para la producción y evaluación formativa de medios instruccionales, aplicado al video y al software.Comunicación presentada al IV Congreso da Rede Ibero Americana de Informática Educativa. Congreso llevado a cabo en RIBIE 98, Brasil.

Ebner, M., Schönhart, J., \&Schön, S. [2014]. Experiences with iPads in Elementary School. Profesorado: Revista de currículum y formación del profesorado 18[3], 161-173. 
Galán, E. [2006]. El guion didáctico para materiales multimedia. Espéculo. Revista de estudios literarios [34].

Galvis, A. [1992]. Ingeniería de Software Educativo. Colombia: Ediciones Uniandes.

González Castañón, M. A. [s.f.]. Evaluación de software educativo: orientaciones para su uso pedagógico. Colombia: Universidad EAFIT. Recuperado de http://www.tecnoedu.net/lecturas/ materiales/lectura27.pdf

Marquès, P. [2002]. Evaluación y selección de software educativo. Comunicación y pedagogía: Nuevas tecnologías y recursos didácticos, 185, 31-37. Recuperado de https://dialnet.unirioja. es/servlet/articulo?codigo=306631

Marqués, P. [2007]. Pere Marques. Barcelona: Pere Marquès \& tecnologia educativa. Extraído de http://peremarques.net/ .

Marquès, P. [2012]. Impacto de las TIC en la educación: funciones y limitaciones. 3ciencias. Revista de investigación [3]. Recuperado de https://www.3ciencias.com/wp-content/ uploads/2013/01/impacto-de-las-tic.pdf

Martín Monje, E., Arús Hita, J., RodríguezArancón, P., \& Calle Martínez, C. [2014]. REALL: Rubric for the evaluation of apps in language learning. Madrid, España: Universidad Complutense de Madrid.

Martínez, F., Prendes, M., Alfageme, M., Amorós, L., Rodríguez, T. y Solano, I. [2002]. Herramienta de evaluación de multimedia didáctico. Revista Píxel-Bit. Revista de Medios y Educación [18], 7188. Recuperado de https:/ / dialnet.unirioja.es/servlet/articulo?codigo=1399633

Muñoz, J. [2017]. Apps para músicos. Recuperado de http:/ / www.appsparamusicos.com/

Pastor, J. (18 de marzo del 2014). Desarrollo de aplicaciones móviles (II): ¿Para qué plataformas móviles desarrollar y para cuál primero? [Mensaje en un blog]. Recuperado de https://www. xatakamovil.com/mercado/desarrollo-de-aplicaciones-moviles-ii-para-que-plataformas-movilesdesarrollar-y-para-cual-primero

Reyes Caballero, F., Humberto Fernández Morales, F., Duarte, J. E. [2015]. Herramienta para la selección de software educativo aplicable al área de tecnología en educación básica. Redalyc. Red de Revistas Científicas de América Latina y el Caribe, España y Portugal, 11 [1], 186-193. Recuperado de http:/ / www.redalyc.org/articulo.oa?id=265440664013 
Rial Rebullido, T., Villanueva Lameiro, C. [2013]. Las aplicaciones para terminales móviles como herramienta didáctica en el desarrollo de contenidos rítmicos y expresivos. EmásF: revista digital de educación física, 4 [23], 7-15. Recuperado de https://dialnet.unirioja.es/servlet/ articulo?codigo $=4483108$

Richmond, F. [2015]. Learning music with GarageBand on the iPad: The essential classroom guide to music production, performance, and education with iOS devices. Van Nuys, California: Alfred Music.

Rudolph, T., Leonard, V. [2014]. Musical iPad®: Performing, creating, and learning music on your iPad®. Milwaukee, Wisconsin: Hal Leonard Books.

Smith Pascuas-Rengifo, Y., Owaldo Vargas-Jara, E., Sáenz-Núñez, M. [2015]. Tecnologías de la información y las comunicaciones para personas con necesidades educativas especiales. Redalyc. Red de Revistas Científicas de América Latina y el Caribe, España y Portugal, 11 [2], 240-248. Recuperado de http:/ / www.redalyc.org/articulo.oa?id=265443638018

Soto, F., Gómez, M. [2002]. EVALÚA: Un instrumento de evaluación de recursos multimedia para la atención a la diversidad. Recuperado de http://diversidad.murciaeduca.es/tecnoneet/ docs/2002/5-22002.pdf

Squires, D., McDougall, A. [1997]. Cómo elegir y utilizar software educativo. Madrid: Morata.

Tortosa Nicolás, F. [2004]. Proyecto: TIC for TEA. Murcia, España.

Watkins, A. [2001 ]. Aplicación de las Nuevas Tecnologías a las Necesidades Educativas Especiales. Últimas tendencias en 17 países europeos. Middelfart: European Agency forDevelopment in SpecialNeedsEducation. 